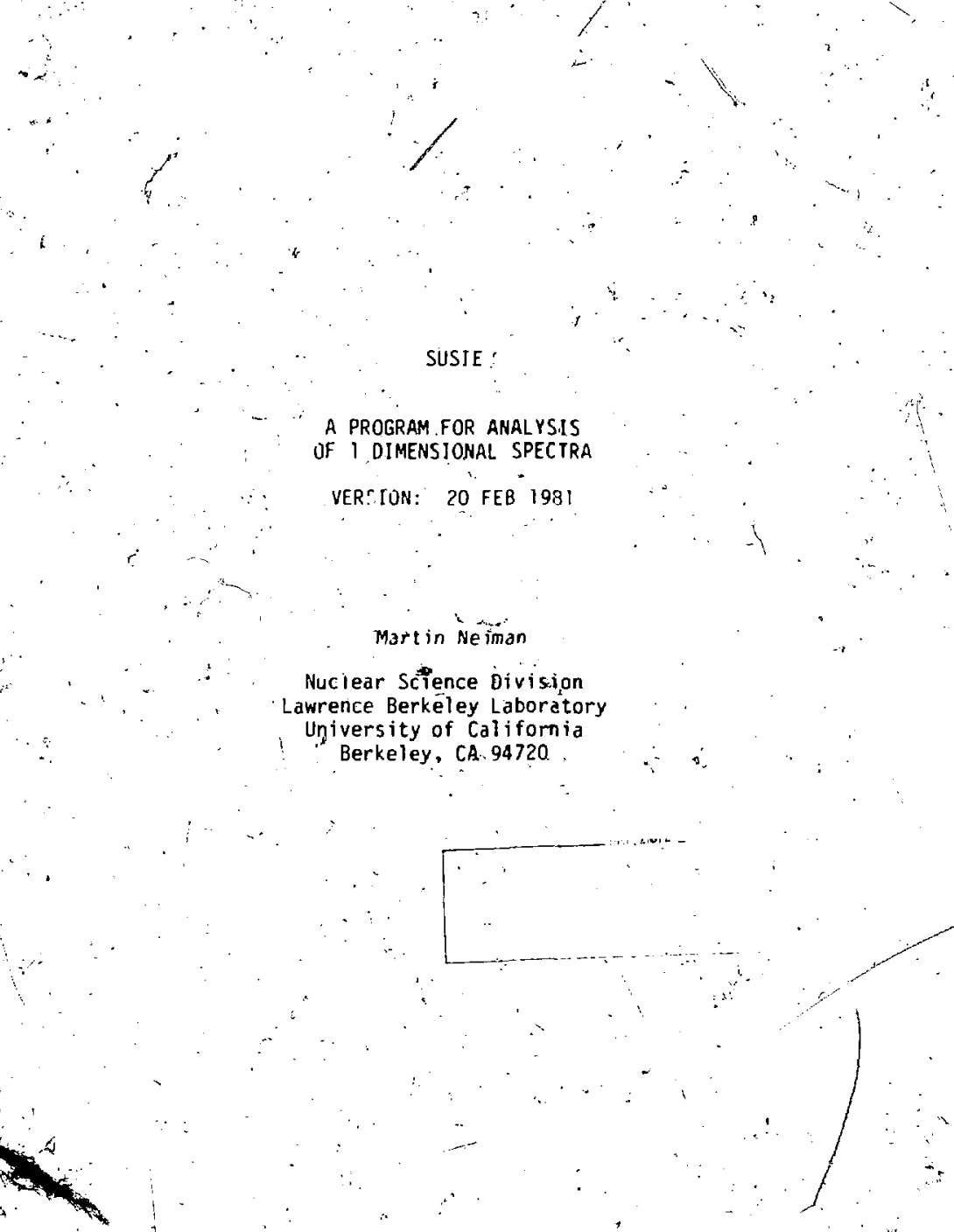

VER ION: 20 FEB 1981

Mart in Neiman

Nuclear Science bivision Lawrence Berkêtey Laboratory

niversity of California

Berkeley, CA. 94720.

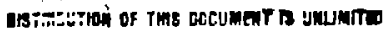

- This work was supported by the U.S. Department of Energy under Contract No. W-7405-ENG-48. 


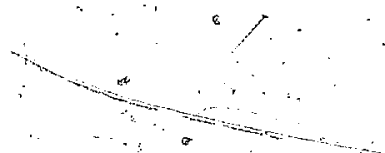

INTRODUCTION

1 Susie is a program for analysis, of 11 dimensional spectra. "It uses a disk for spectrum storage, only: keeping one spectrum (the current esiectrum); in core at a time. This enables' Susie to manipulate 20 spectra, numbered fromi 1 to 20 . A.t. commands operate upon the current spectrum. There are commands to fit peaks and otherwise manipulate the data in the spectra as well. as commands to move spectra both to and from the disk and to and from magnetie tape.

Following this introduction there is a list: of comands, followed by a more detailed description ofl each individual comand. Finally there is a.section with some practical information, and an example of a brief session. using Sus ie.

In this manual I have used the following notational convientions:

1) Parentheses indicate opt ional parameters.

2) Underlining means that the text string, rather than a numeric value, is wanted.

All text strings may be aboreviated by us ing on?; the first four letters: 


\section{LIST OF COMMANOS}

A 90

SPECTRUM, FIRST SPECTRUN (;LAST SPECTRUM)

REAL NUMBER

CHANGE OLD, NEW

COMPRESS CHANNELS

BIVIDE : SPECTRUM, SPECTRUM NUMBER

REAL NUMBER

EXIT

EXPANO (LUW, HIGH)

FIT : :

GET SPECTRUM NUMBER

$\frac{\text { INPUT }}{5} \ldots$ (AXQ)

LABEL

$\underline{L I N}$

(ALL)

$\{06$

NUMBER OF CYCLES) (, ALL)

MAKE. FUNCTION, $+1 ; A 2$

MAP

(FIRST, LAST)

MARK

(CHANNEL).

MUNENTS (LOW, HIGH)-

MULTIP $\triangle$ SPECTRUM, "SPECTRUM NUMBER

REAL-NUABEP

SPECTRIMM NUMBER (, NUMBER OF CHANNELS)

$\therefore \quad$ ALL (, MUMBER OF CHANNELS)

PLUT $\quad$ (FLATBED)

PRINT (LOW, HIGH)

READ $\therefore$ SPECTRUM NUMBER, TAPE FILE (: RELATIVE, NUMBER IN FILE) (. SKIP, NUMBER OF. CHANNELS)

RESTORE (ALL)

SAYE $\because$ SPECTRUM NUMBER 


\section{SCALE. (FULL SCALE) \\ $\therefore$ SET CHANNEL, CONTENTS \\ SHIFT : CHANNECS \\ SİZE NUMBER OF CHANNELS (; ALC)}

\section{SMOOTH}

SUBTRACT SPECTRUM, 'SPECTRUM NUMBER

REAL NUMBER

SUM

(LOW, HIGH)

TAPE

UNIT (NEWTAPE)

WRITE

(FIRST SPECTRUM) (; LAST SPECTRUM)

ZERÓ. . (LOW, HIGH)

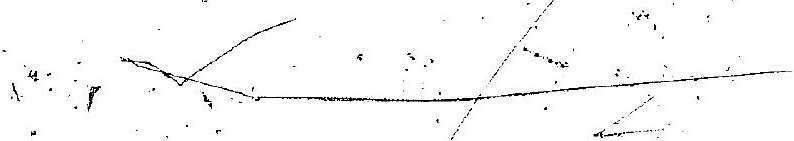


F

(L)

or

ADD

R

The if irst form will add spectrum number $F$ to the current spectrum. If the optional parameter $L$ is given, all spectra from $F$ to $L$ will be added. io the current spectrum. The second form will add the real constant $R$. channel by channel, to the current spectrum. The channel contents are interese. co the sum is rounded to an integer.
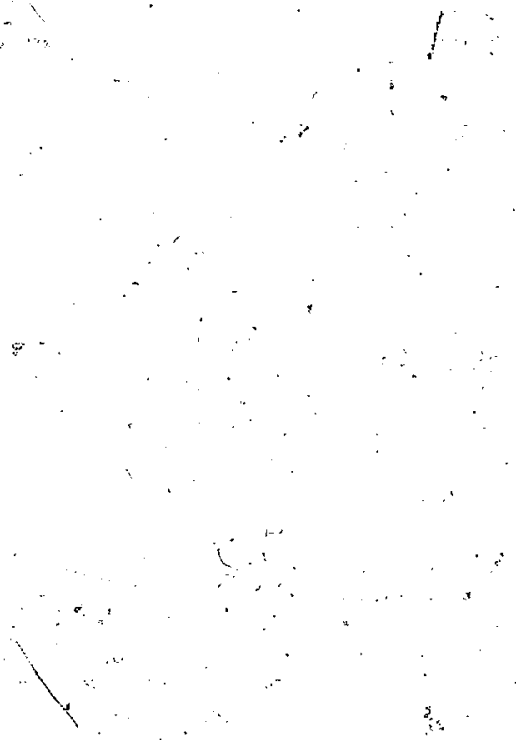
Change will scan the current spectrum, channel by channel. If any channels. are found which contain OLO counts, the contents of that channel is changed to NEW.

\section{EXAMPLE}

(स)
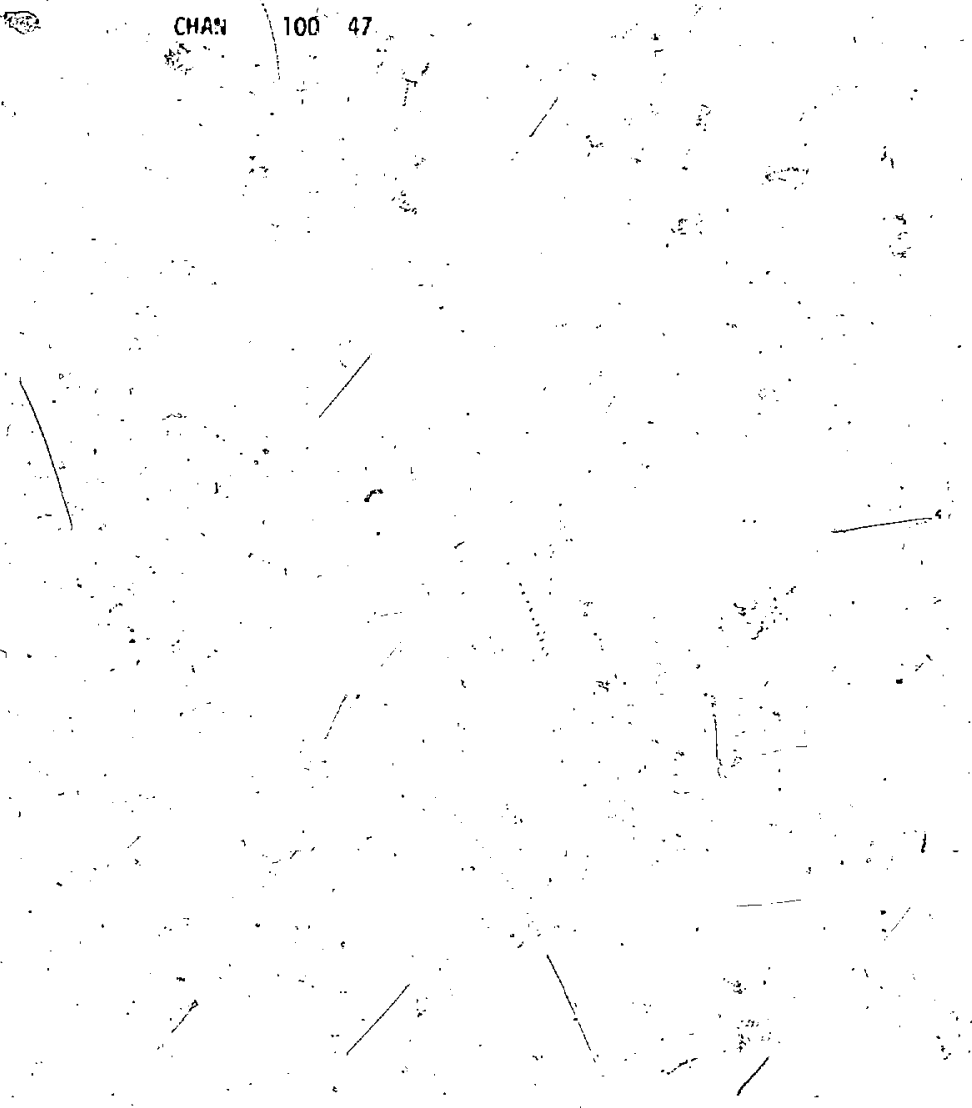
COMPRESS $\ddot{C}$

Compress will take the current spectrum and compress it by adding $C$ channels together. If $C$, is a real number small rounding errors may result.

EXAMPLE

$$
\because \quad \text { COMP } \quad 100
$$


DIVIDE - SPECTRUM $S$ or

DIVIDE $\because \frac{6}{k}$

The first fom will divide the current spectrum by spectrum number $S$ : Zero channels in spectrum $\$$ are treated as follows: If the corresponding channel fin the currer, spectrum contains zero, the resulf is set to l; otherwise, the result is set to the arbitrary value 10,000. The second fom will divide, the current spectrum, channel by channel, by the real number $R$. The result is ro inded to an integer.

EXAMPLES

OIVI! SPEC 10

0] VIDE . 28.3 
ExIT

This command is used to terminate the program.

ExIT

y

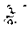

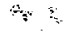

Ex. 


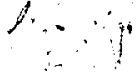

EXPAKD

EXPAND (LOW HIGH)

This comand is used to select an interval of the current spectrum and expand it so that it fills the entire width of the display screen." If. the optional paraneters LOW and HIGH are given, the interval from LOW. to HIGH will be displayed. If they are absent, a cursor will, apear and is used to inicate the desired interval.

\section{EXAMPLES}

EXPA : 123550

EXPAND
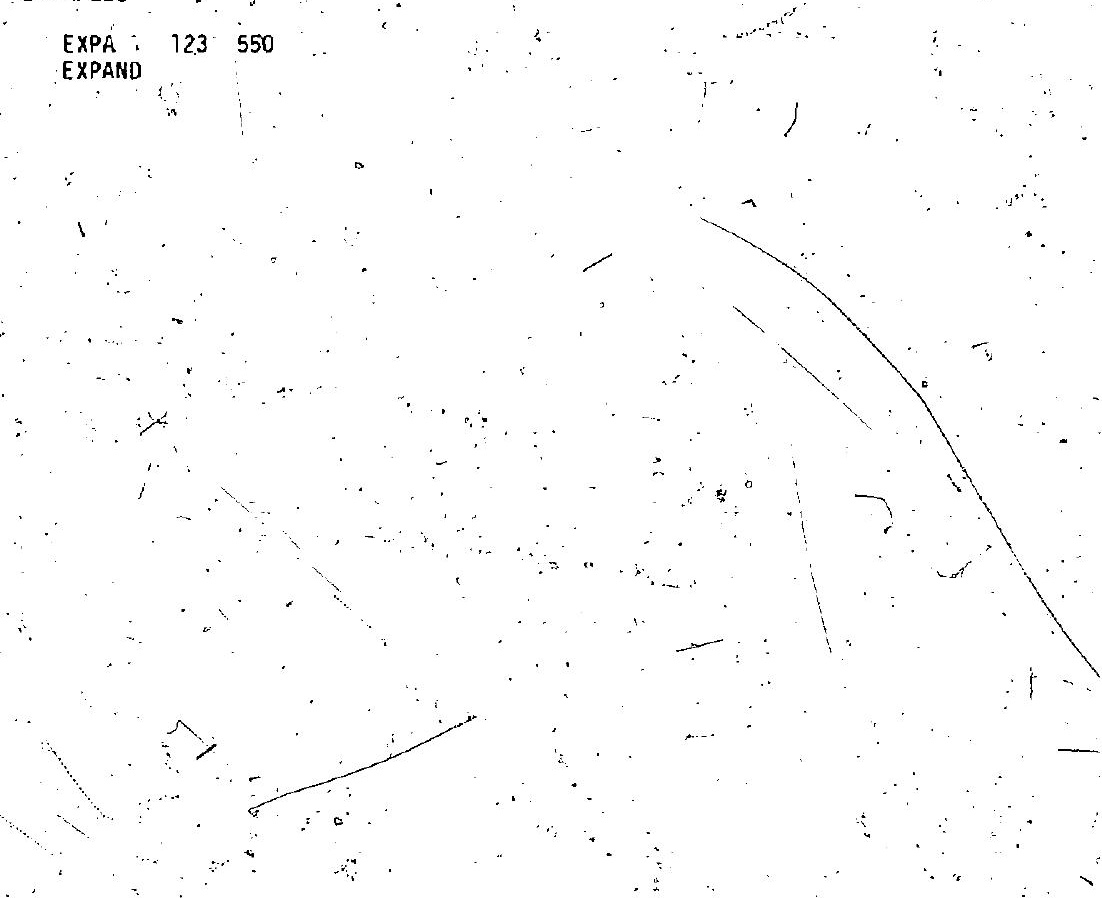
FIT (FUNC)

where FUNC may have the value: PEAKS, EXPONENTIAL, or LINE.

This command is used to $f$ it a function to an interval of the current spéct rum.

If FUNC = PEAKS (or is absent), the funct ion is a linear background plus 1 to 3 Gaussian peaks (all of the same width).

The function used is

$$
\because \quad Y(x)=A_{1}+A_{2} x+A_{4} e^{-\frac{1}{2}\left(\frac{x-A_{5}}{A_{3}}\right)^{2}}+A_{6} e^{\frac{1}{2}\left(\frac{x-A_{7}}{A_{3}}\right)^{2}}
$$

First the interval to be $f$ it is indicated, using the cursor. Then the peak positions are indicated with the cursor., One indicates that the cursor is - in the desired position by hitting a key on the Tektronix-keyboard. If a space is used, the cursor will, remain on the screen so that additional peaks can be indicated. Any key other than a space will initiate the fitting.

If FUNC = EXPONENTIAL the function used is

$$
Y(x)=A_{1} e^{A_{2}{ }^{x}}
$$

The intervat to be fit is indicated us ing the cursor.

If -FUNC = LINE the function used is

$$
Y(x)=A_{i} x+A_{2}
$$

The interval to be $f$ it is indicated using the cursor.

\section{EXAMPLES}

FIT

FIT PEAK

FIT EXPO

FIT ' LINE 
GET $S$

This command will get spectrum $S$ from the disk, display it, and make it the current spectrum.

EXAMPLE

$$
\text { GET } \quad 12
$$




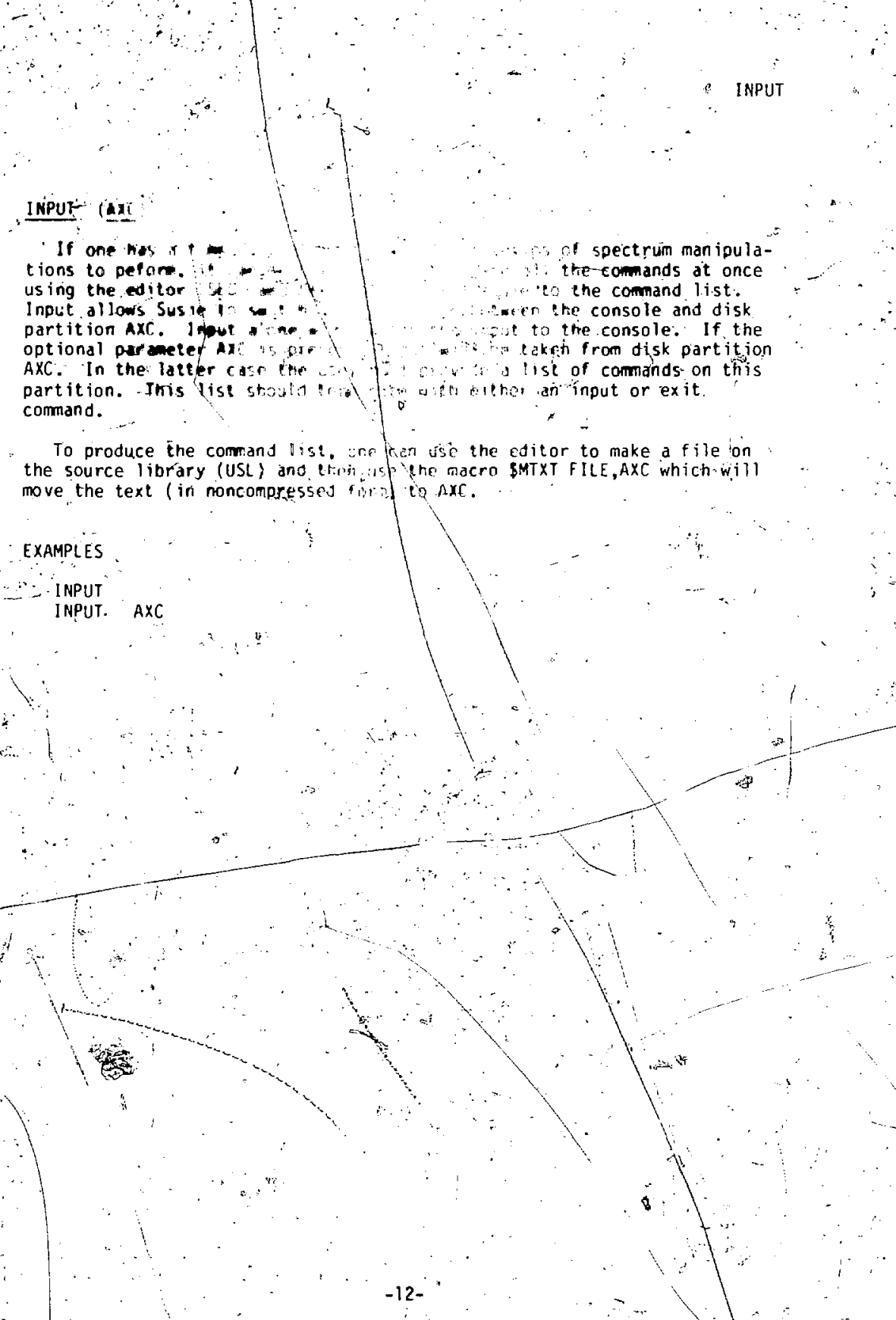


LABEL

This comand will request a 32 character label. The label will be

$\therefore \quad$ dicplayed on the screen and be printed out when a spectrim is printed on

$\therefore \quad$ dicplayed on the screen and be printed out when a spectrim is printed on 1
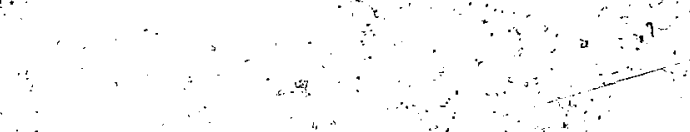

\section{$\rightarrow$}

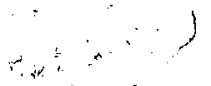

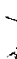

$3^{2}$

$\ldots$

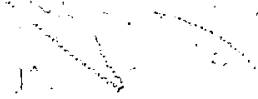

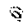
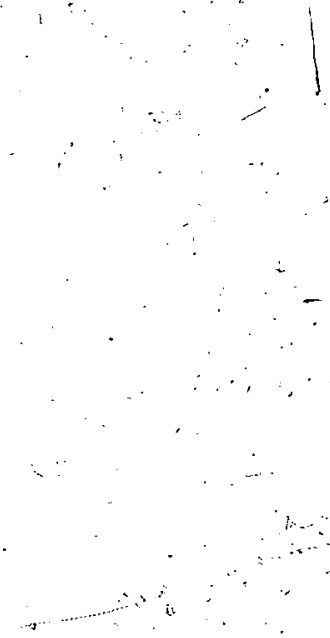

$z^{\prime}$ 
$\underline{\operatorname{LIN}} \cdot(\underline{A L L})$

- This command will change the display of the current spectrum to linear $\because$ in the $Y$ axis. If the optional parameter ALL is given, all spectra, when. - they are displayed, will be in linear form.

- EXAMPLES .

$$
\begin{aligned}
& \text { LIN } \\
& \text { LIN }
\end{aligned}
$$

ALI

$y^{3}$

LIN

$\rightarrow$

a 


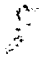

LOG. (N) (ALL)

This command will change.the display of the current spectrum to semilog. If the aptional parameter $N$ is given, the spectrum will be displayes with $N$ cyćles. N may assume values from 2 to 9 . If $N$ is omitted (or. zero) the number of: cycles will automatically adjust. If the optional parameter ALL is given, all spectra, when displayed, will be semi-licg.

EXAMPLE'ES

LOG

LOG

$\angle O G$

LUG

$$
\dot{3}
$$

ALL

A. ALL 
MAKE FUNC Af A 2

Where FUnC may have the value: EXPONENTIAL, LINEAR.

This command will produce. a spectrum containing a calculated function. Uniy the interval of the spectrum displayed will contain the function, the. remainder being set to zero: If FUNC = EXPONENTIAL the finction used is

COUNTS $(\mathrm{CH})=\mathrm{A}^{\star} \mathrm{E} \times \mathrm{P}\left(\mathrm{A} \mathrm{A}^{\circ} \mathrm{CH}\right)$

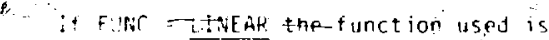

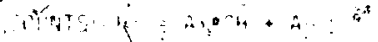

$\dot{\beta}_{x} d x$

in. $\quad \therefore$

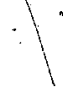

$\because+i, \ldots$

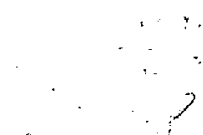

$i^{4} \quad j$

$\frac{32}{2}$

$\therefore \sin 0^{\circ}$
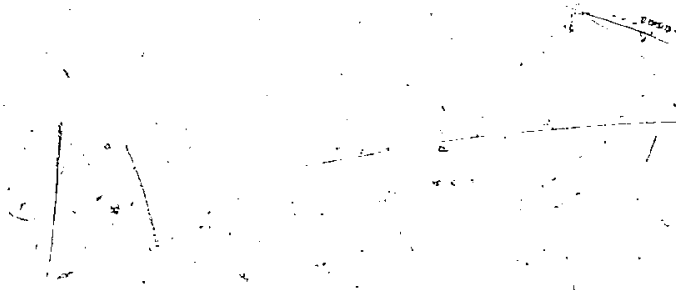

trats 
MAP (FTRST, LAST)

This command" will. print a map of the input spectrum tape on the line printer. The map will show the contents and titles of ail files. If the optional parameters FIRST and LAST aregiven, onty the interval frof FIRST to LAST will be printed.

$\pi: 5$
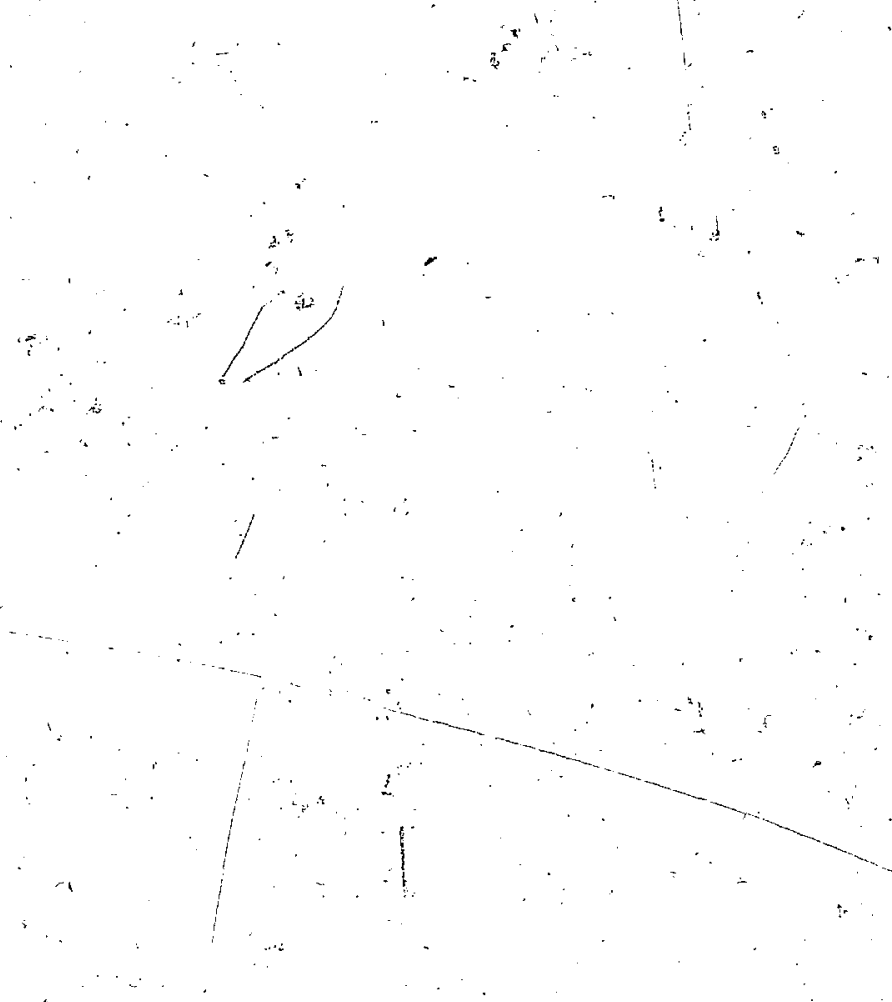

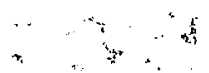

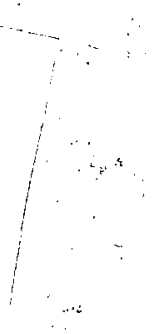




\section{MARK (C)}

This commans is used to mark a channel in the current spectrum. If the optional parameter $C$. js iven, that channel will be marked on the display.: If no channel is given, the cursor will appear and is ûsed to indicate the channel jesired, and the number and contents of the channel are printed. if the cursor position is indicated by typing a space, the cursor will ieturn and another channel tan be marked. Any character other than a space wil terminate the comand.

\section{Iximpis}

ising , in: :
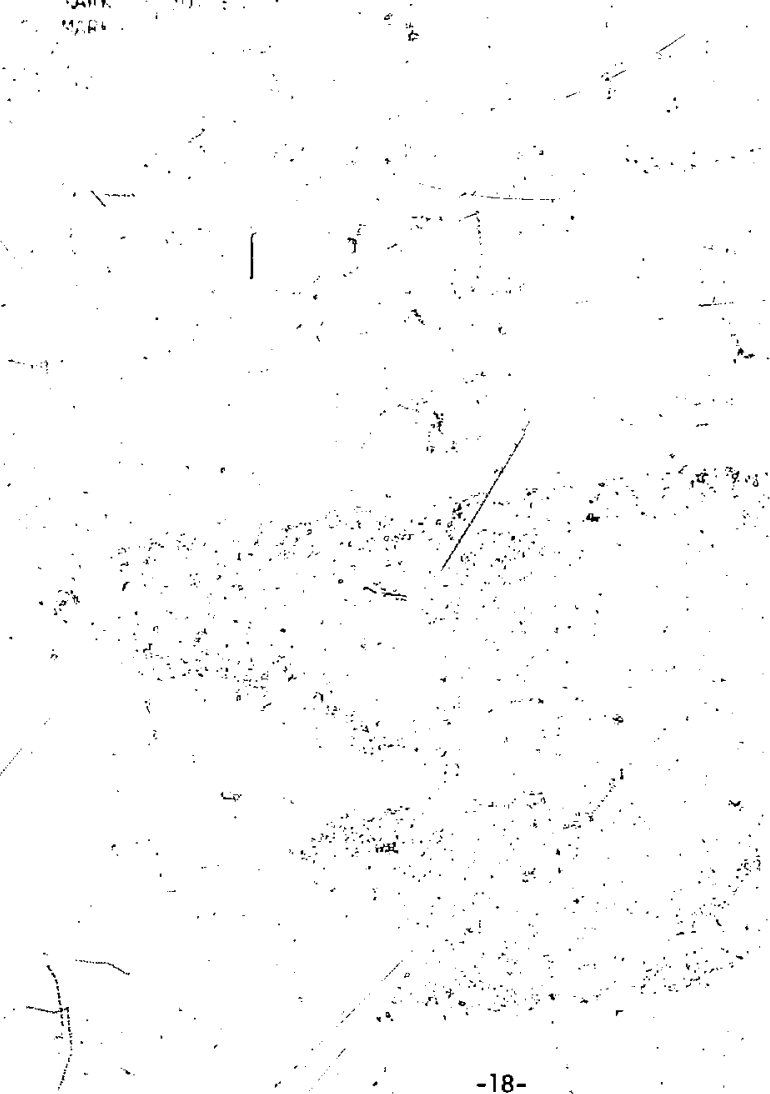
MOMENTS

MOMENTS (ION HIGH)

iris comiand will define an interval of the current spectrum and calculate the first three moments of the distribution of counts in that interval. If $\mathrm{LOH}$ and $\mathrm{HIGH}$ are given, they are used to define the interval; otherwise, the cursor appears and must be used, The moments are defined as follows:

- CENTR010: $\cdot \frac{1}{1}=\langle x ;$

VARiances: $?=x^{?}-x^{2}$

$$
x^{3}-3 \cdot x^{2} \ldots+2 \cdot x^{3}
$$

EXAmPLES

$$
\begin{aligned}
& \text { HOME } 100 \text { SO } \\
& \text { MOMENTS }
\end{aligned}
$$

$\because$

$-19-\$$ 
i.

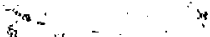

MULTIPLY

से

the

MULTJPLY SPECTRUM $S$, or

MULTIPLY

The first form will multiply the current spectrum by spectrum humber $S$. The second form will multipty the current spectrum, channel by channel, by the real constant. $R$. The result is rounded to an integer.

\section{EXAMPLES}

MULT SPEC 9

MULT: 14,42 .

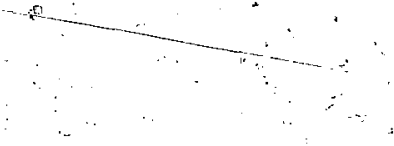


OLO SPEC (NUMBER OF CHANNELS)

ALL (NUMBER OF CHANNELS)

The spectra in' Susie are stored on thë upper disk. (on partition RSW). This command tells Sus ie to use the data on the disk as spectra. The first form of the command def ines spectrum SPEC as existing on the disk. The second form defines all spectra as existing. If the optinal parameter NUMBER OF CHANNELS is given; the spectra are def ined to have that length; otherwise, the spectra are assumed to be the maximum size (defined when Sus ie is called).
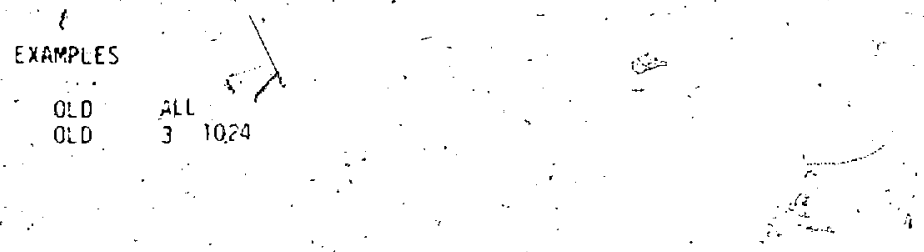


\section{PLot (Flatbed)}

This command will make a plot of the current spectrum. Foli scale, LJN/LOG mode, and the interval of the spectrum plotted will be the same "as seen on the display. If the optional parameter FLATBED is given,- the plot will be an exact duplicate af the screen plotted on the Tektronix flatbed plotter. Qtherwise, the plot will appear on the Versatec printer. There will be short tic marks every 10 channels and longer tic marks every 100 Channels.

PLOT

PLOT

$$
\text { Fis: }
$$

$$
\text { i) }
$$




\section{PRINT (LOW, HIGH).}

This command will print a listing of the current spectrum on the line printer. If the optional parameters Low and HIGH are given, on $y$ the interval from low to high ill be printed.

\section{EXAMPLES}
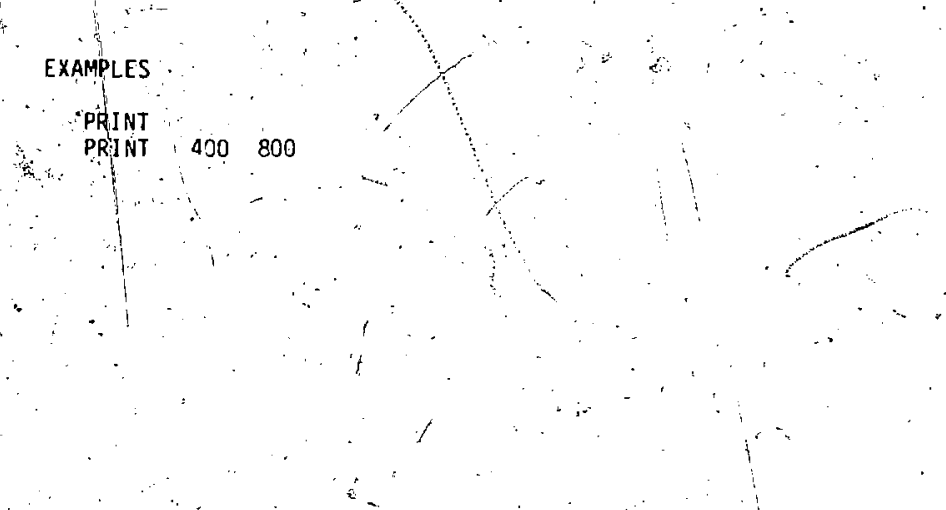
$\underline{R E A D}$ 'S $F$ (N) (SKIP, C)

This command is used to redd spectra from tape. It will.read one: spectrum from file. F. make it the current spectrum, and place it on disk as spectrum number $S$. If there is more than one spectrum in file $F$, the . Nth spectrum is read. If $\mathrm{N}$ is omitted, $\mathrm{N}=1$ is assumed.

If the optiona? parameters SKIP and $C$ are present, the first $C$ chan of the spectrum, will be skipped before starting to read.

EXAMṔLES

RERE

READ $3 \quad 47^{\circ} 4$

$\therefore$ REAT 12 9 SKIP 190

RERD :20:3. 2 SKIP 512

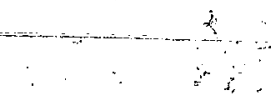


This comiand will restore the display of the current.spectrum, so that the entire spectrum is displayed. If the optional parameter ALL is given all spectra will be restored (when they are displayed).

EXAMPLES .

REST

REST

ALL

F.
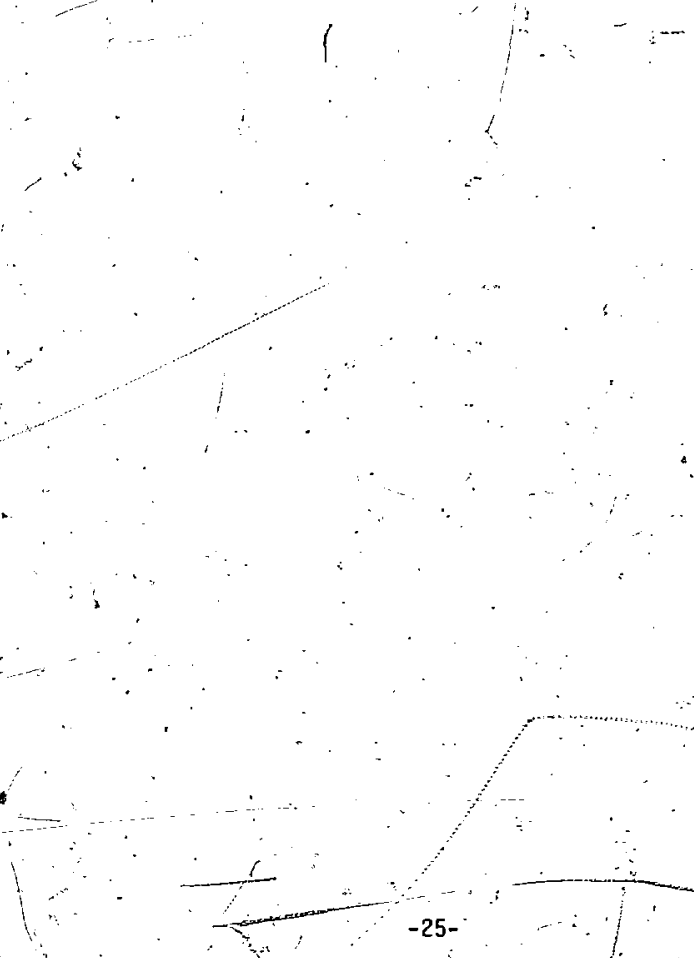
SAVE I S

This command saves the current spectrum on disk as spectrum number $S$.
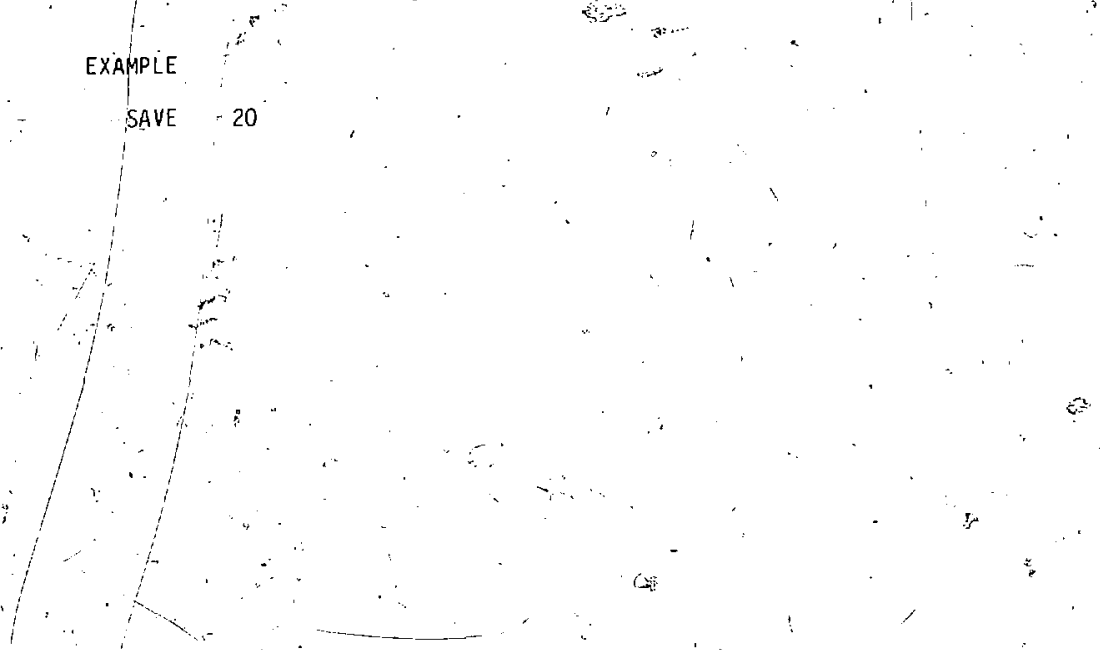

s

p. 
SCALE (F)

This command is used to change the full scale of the display of the current spectrum. If the notional parameter $F$ is given the full scale is set to F! otherwise, the cursor appears and is used to indicate the full scale desired.

EXAMPLES

$$
\text { SCALE } 2500
$$

a.

33

$-27-$ 
This command is used to change the contents of one channel in the current spectrum. It will take channel number $C$ and insert $k$ counts in it.

EXAMPLE:

SET" $510{ }^{\circ} 23492$

$\because e^{2}$
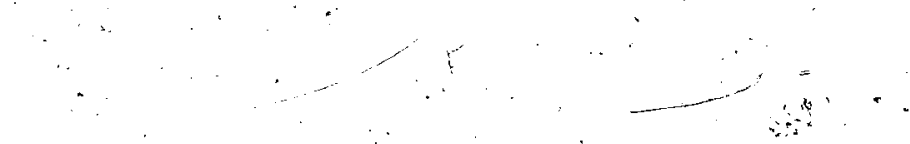

$\div$

\section{药}

i)

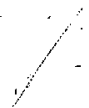


$\therefore$

$\therefore \quad$

\section{SHIFT CHANNELS}

Shifts the current spectrum by CHANNELS. The channels shifted out are lost, and zeros are shifted in. If channels, 0 the spectrum is shifted to the right; if channels 0 the shift is to the left.

\section{EXXMPLES}

SHIFT 3

SHIF: -5
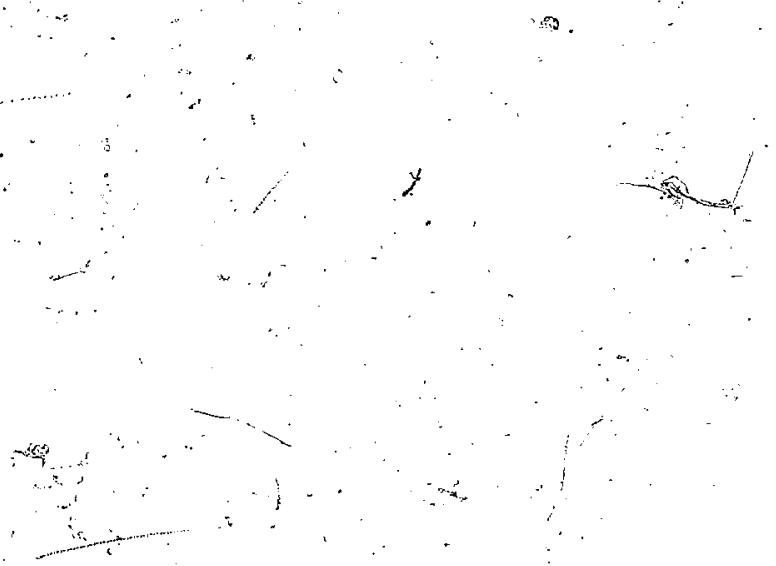
SIZE: N. $(\underline{A L L)}$

1

SIZE

Size will change the size of the current spectrum to $N$ channels. If the optional parameter ALL is given, all spectra will be changed to $\mathrm{N}$ channels.

EXAMPLES

$\begin{array}{ll}\text { SIZE } & 512 \\ \text { SIZE } & 1024\end{array}$

$i$

$\because$

i

$\therefore$

$\therefore$ 
SMOOTH

SMOOTH

This command will. smooth the current spectrum using a 3 channel interval.. "The formula used is:

$$
\text { i.". }
$$

$$
\because
$$$$
\text { SOOTH. }
$$

$x^{2}$

$\therefore \quad i$

.

$\begin{aligned} \mathrm{Y}^{\prime}(\mathrm{CH}) & =\frac{1}{4} \mathrm{Y}(\mathrm{CH}-1)+\frac{1}{2} \mathrm{Y}(\mathrm{CH})+\frac{1}{2} \mathrm{Y}(\mathrm{CH}+1) \cdot 5 \\ & =\cdots\end{aligned}$ 
The first form will subtract spectrum number $S$ from the current spectrum. The second form will add real constant $R$, chaninel by channel, to the current spectrum. The resul.t is rounded to an integer.

\section{EXAMPLES}

\section{SUBT SPEC . 1}

SUBT: $\quad 100$

80

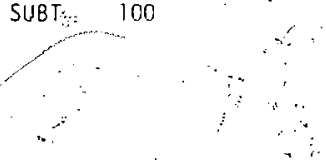

3

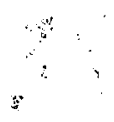

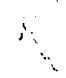




\section{SUM (LOW HIGH)}

This commans will defire, an interval of the current spectrufin and sum the counts in the interval. If the optional parameters LOW and HIGH are given, they are used to define the interval; otherwise, the cursor appears, and must be used. The interval includes the end points.

\section{EXAMPLES}

\section{SUM}

$100 \quad 450$

SUM
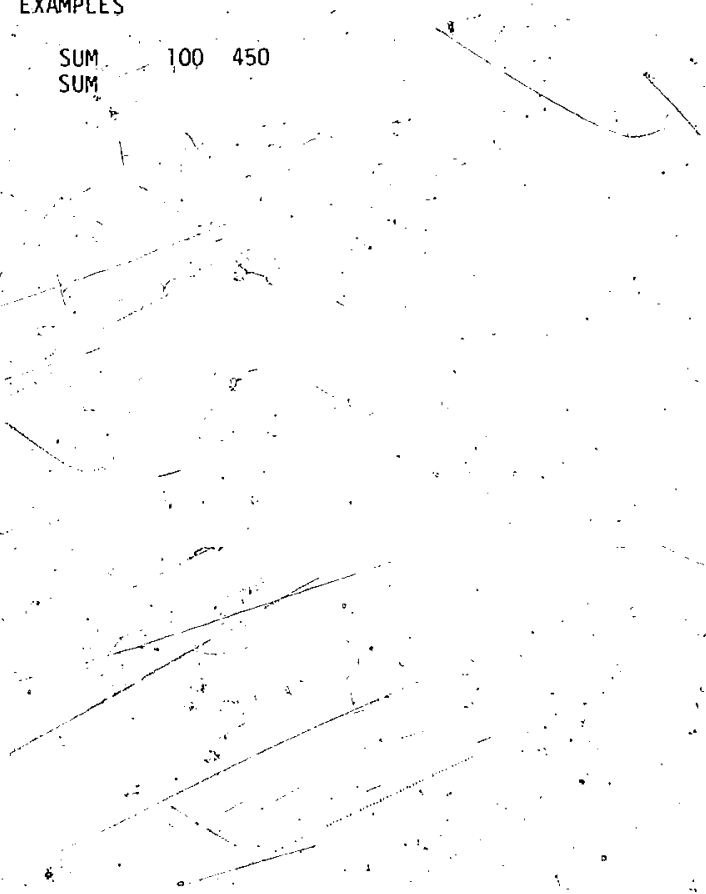
TAPE: UNIT (NEWTAPE)

This command defines the tape station used. The al lowate values for UNIT: are 1, 2, 9 indicating MT1; MT2, MT9 respestively. If the optiona? parameter NEWTAPE is given, the tape is assumed to be a new tape meaning that spectra will be written directly at the beginning of the tape:

\section{EXAMPLES}

$\begin{array}{ll}\text { TAPE } & 2 \\ \text { TAPE } & \text { NEWT }\end{array}$

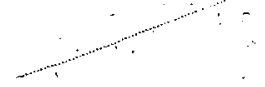

18 
WRITE (F) (L)

This command ismused to write spectra on tape. If no parameters are "given, the current spectrum is written. "If the optional parameter $F$ is given alone, spectrum number $F$ will be made the current spectrum (but not Gisplayed), and written on tape. 'If both $F$ and.L are specified, spectra numbers. $F$ through $L$ will be written. Each spectrum will be writter on its own $j$ le. A tit le: (maximum 32 characters) will be requested.

\section{EXAMPLES}

WRIT

$\therefore . \cdots$
$\therefore$.

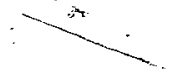

\section{WRLTE}

, WRIT. $5 . \cdot 14$

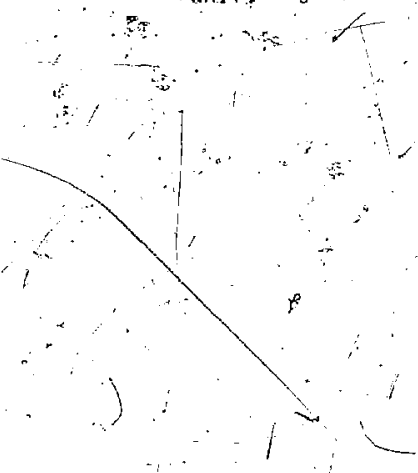




\section{IERO}

ZERO LOW HIGH

Sets the contents of all channels in the inclusive interval from Low to HIGH to zero.

EXAMPLE Q

ZERO "1000" "1023:"

w.

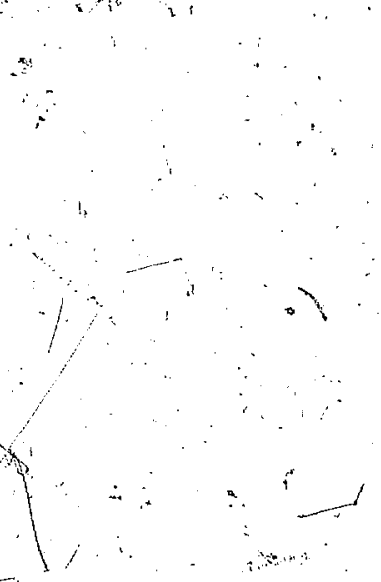

(1)
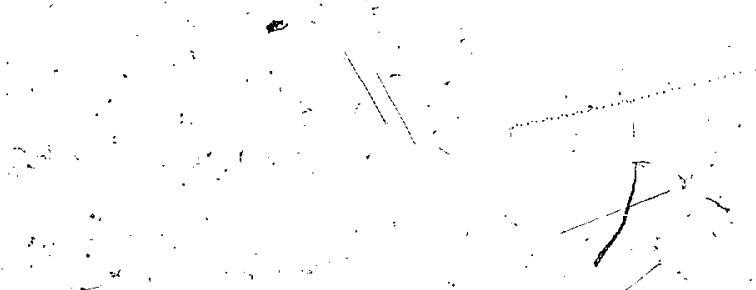
